\title{
Tendencias en la evaluación del desarrollo y la gestión del conocimiento
}

\author{
Trends in development evaluation and implications \\ for knowledge management
}

\section{Osvaldo N. Feinstein ${ }^{I}$}

I Profesor del Máster en Evaluación de la Universidad Complutense de Madrid. Exgerente del Departamento de Evaluación de Operaciones del Banco Mundial.

$\bowtie$ ofeinstein@yahoo.com https://orcid.org/0000-00016144-0614

Este artículo fue publicado en inglés, en 2017, en Knowledge Management for Development Journal, 13(1), 31-38, http://journal.km4dev.org.

Versión en español revisada por el autor.

\section{Resumen}

Este documento identifica las tendencias en la evaluación del desarrollo que surgieron durante la última década, como el uso de ensayos de control aleatorios, el renovado énfasis en la complejidad, la creación de redes y asociaciones de evaluación nacionales, regionales y mundiales, el creciente uso de las TIC (tecnologías de la información y el conocimiento) para el monitoreo y las prácticas de evaluación, y el interés en utilizar la evaluación como insumo para la toma de decisiones a mayor escala. El documento considera oportunidades y riesgos asociados con estas tendencias y propone formas de abordarlas, incluyendo un papel clave para la gestión del conocimiento.

Palabras clave: gestión del conocimiento, futuro de la gestión del conocimiento, aprendizaje, ensayos de control aleatorios, complejidad, evaluación, difusión, tecnologías de la información y el conocimiento.

\section{Abstract}

This paper identifies trends in development evaluation that emerged during the last decade, such as the use of randomized control trials, the renewed emphasis on complexity, the creation of national, regional and global evaluation networks and associations, the increasing diffusion of ICTs in monitoring and evaluation practice and the interest in using evaluation to inform scaling-up 
decisions. The paper considers opportunities and risks associated with these trends, proposing ways to deal with them, including a key role for knowledge management.

Keywords: knowledge management, future of KM, learning, RCT, complexity, evaluation, diffusion, ICT.

\section{Introducción}

En este documento se identifican las principales tendencias de la evaluación del desarrollo y se examinan sus consecuencias para el futuro. En lugar de especular sobre escenarios futuros, que en un contexto de gran incertidumbre pueden resultar muy dispares, se sigue el enfoque del futuro sugerido por Peter Drucker, ${ }^{1}$ que examina importantes tendencias que ya han surgido y explica en detalle cómo hacer frente a los desafíos que estas generan. Las tendencias de la evaluación del desarrollo que se tendrán en cuenta son las siguientes: el uso de ensayos de control aleatorios, un énfasis renovado en la importancia de la complejidad, la creación de redes y asociaciones de evaluación nacionales, regionales y mundiales, la creciente aplicación de las tecnologías de la información y la comunicación (TIC) en la práctica de la evaluación, y el interés en utilizar la evaluación para fundamentar la toma de decisiones a mayor escala. Cabe señalar que estas tendencias han sido desencadenadas por diferentes fuerzas e involucran a diferentes actores, por lo que, al menos hasta ahora, aparecen bastante desconectadas.

En el documento también se analizan los vínculos entre la evaluación del desarrollo y la gestión del conocimiento. Se concluye con un examen de las oportunidades y los riesgos asociados a las tendencias identificadas, y se proponen formas de abordarlos, resaltando el papel clave de la gestión del conocimiento.

\section{Tendencias importantes}

En lugar de sugerir tendencias futuras no ancladas en las realidades actuales, este documento se enfoca en las tendencias actuales más importantes, que probablemente continuarán y se ampliarán en el futuro. ${ }^{2}$

$1 \quad$ Véase nota 3.

2 Nos recuerda al poema de Burnt Norton de T. S. Eliot:

Tiempo presente y tiempo pasado.

Quizás ambos estén presentes en el tiempo futuro

y el tiempo futuro contenido en el tiempo pasado. 


\section{Mayor uso de ensayos de control aleatorios (ECA)}

El uso de ensayos controlados aleatorios (ECA) en la evaluación del desarrollo tiende a consolidarse tanto por el lado de la demanda como por el de la oferta, a pesar de las críticas realizadas principalmente por evaluadores profesionales, y de la opinión de que los ECA son una burbuja condenada a estallar (Picciotto, 2012). Instituciones financieras internacionales como el Banco Mundial y el Banco Interamericano de Desarrollo, así como la Fundación Gates, entre otras, están apoyando y proporcionando fondos para la realización de ECA, y organizaciones muy activas, con profesionales altamente competentes, como 3ie y el JPAL del MIT también los están llevando a cabo. Más aún, JPAL ha estado implementando un programa de capacitación mundial centrado en los ECA.

Además, las principales revistas económicas, como la American Economic Review, están publicando artículos que informan sobre los resultados de los ECA. Esto constituye un incentivo adicional para asignar recursos a este enfoque, y aunque ha habido algunos estudios que muestran las limitaciones de los ECA y la necesidad de un enfoque más amplio (Stern et al., 2012), así como la crítica de eminentes economistas (Deaton, 2010), existe un consenso generalizado entre los formuladores de políticas (aunque no entre los evaluadores) de que los ECA son el enfoque científico de la evaluación, el patrón oro de la evaluación. Esto demuestra la influencia que ejercen las organizaciones de financiación, así como el número cada vez mayor de profesionales capacitados en la realización de ECA, sobre los encargados de la formulación de políticas. Por otra parte, es importante tener presente que los ECA deben utilizarse junto con otros enfoques, precisamente para dar explicaciones sobre los resultados observados. ${ }^{3}$

\section{Énfasis renovado acerca de la importancia de la complejidad}

La evaluación del desarrollo prácticamente comenzó con la evaluación de proyectos de desarrollo rural integrado, en la década de 1970. Se trataba de proyectos que a veces tenían hasta veinte componentes interrelacionados, que se percibían como complicados y complejos. Los evaluadores del desarrollo intentaron lidiar con esta complejidad. En parte como resultado de estas evaluaciones, los proyectos se simplificaron, reduciendo drásticamente el número de componentes y la complejidad de las intervenciones.

Cabe señalar que el Premio Nobel en Economía fue otorgado en 2019 a tres economistas que fundaron y lideran el JPAL (Abhijit Banerjee, Esther Duflo y Michael Kremer), y que en 2015 y 2000 fue conferido a Angus J. Deaton y a James Heckman, respectivamente. Deaton y Heckman han sido y continúan siendo muy críticos de los ECA. 
En el siglo XXI, la complejidad volvió a ser una cuestión importante con la intensificación de la globalización, el desarrollo de sistemas adaptativos complejos, la creciente conectividad y la reducción sustancial de los costos de transacción. Las implicaciones de un enfoque complejo de la ayuda están bien presentadas en Ramalingam (2013), y las aplicaciones de este enfoque a la evaluación del desarrollo se presentan en Bamberger et al. (2015) y en Befani et al. (2015).

\section{Interés creciente en el uso de evaluaciones para fundamentar decisiones a mayor escala}

Los gobiernos y las organizaciones internacionales están especialmente interesados en ampliar las intervenciones que han tenido éxito a nivel local. Con una población en expansión en el mundo en desarrollo, es probable que aumente la presión para la ampliación. Además, la mayor disponibilidad de conocimientos sobre lo que funciona, generada por las evaluaciones del desarrollo, brindará oportunidades para identificar intervenciones de desarrollo prometedoras que podrían ampliarse. Sin embargo, se debe tener conciencia de que lo que ha funcionado en un contexto específico puede no funcionar en un contexto diferente, y que la escala es un elemento clave del contexto. El hecho de saber que un proyecto de microfinanzas destinado a tres mil hogares funcionó muy bien en Kenya no garantiza que también tenga éxito, incluso en el mismo país, si la población destinataria es de seis mil hogares; por ejemplo, puede haber una escasez de capacidades de gestión para hacer frente a un grupo objetivo tan amplio, o cualquier otra deseconomía de escala. El conocimiento generado por las evaluaciones puede utilizarse para la ampliación de la escala, pero se debe emplear con cuidado, siendo conscientes de que el éxito a pequeña escala no garantiza el éxito en la ampliación de la escala. También se necesitarán evaluaciones durante el proceso de ampliación para valorar los resultados a una escala de operaciones más elevada. ${ }^{4}$

\section{Creación de redes y asociaciones de evaluación ${ }^{5}$}

Desde finales de los noventa surgieron redes y asociaciones de evaluación en todas las regiones (Feinstein y Beck, 2006). Han desempeñado un papel en el intercambio de conocimientos sobre evaluación y han facilitado el desarrollo de la capacidad para 
evaluar. Aunque la mayoría de esas asociaciones de evaluación tienen su sede en las ciudades capitales, la expansión de la conectividad a Internet (véase el párrafo siguiente) y la implementación de intervenciones de desarrollo en zonas alejadas de las capitales pueden estar llevando a una expansión de esas redes y asociaciones de evaluación involucrando a evaluadores y formuladores de políticas que trabajan a nivel subnacional (incluido el municipal). ${ }^{6}$

\section{Creciente difusión de las TIC en el desarrollo y en la práctica del seguimiento y la evaluación}

Entre las tendencias que pueden redefinir la forma en que se produce el desarrollo global, hay dos de particular importancia para la evaluación del desarrollo: i) la difusión de la conectividad a Internet y la alfabetización digital; y ii) el aprovechamiento de los datos para prestar un mejor servicio a los más pobres y generar nuevos conocimientos. La primera está creando oportunidades para que muchas más personas tengan acceso a la información y, a su vez, la proporcionen, facilitando así la difusión de conocimientos y el flujo de información de abajo hacia arriba, que puede utilizarse para evaluaciones participativas y en tiempo real. La segunda, utilizando herramientas de big data permite la transformación de datos en información y conocimiento (Dervis, 2015).

\section{Vínculos entre evaluación del desarrollo y gestión del conocimiento}

La evaluación genera conocimiento, pero sin acciones específicas existe el riesgo de que este conocimiento no se utilice. Esto se convertirá en un problema aún mayor, ya que con la adopción de las TIC la información y el conocimiento continúan expandiéndose. Se deben tomar medidas para garantizar que el conocimiento se comparta y gestione, de manera que se facilite su uso adecuado para el aprendizaje y la rendición de cuentas de proyectos, programas y/o políticas. La evaluación sin la gestión de conocimiento puede dar lugar a una acumulación de conocimientos no utilizados, mientras que la gestión del conocimiento sin evaluación podría dar lugar a la diseminación de conocimientos no validados sobre intervenciones de desarrollo. Con las nuevas tecnologías, en particular las TIC, la gestión de conocimiento puede facilitar el uso de los 
resultados de la evaluación del desarrollo, tanto para la rendición de cuentas como para el aprendizaje.

En otras palabras, la evaluación sin gestión del conocimiento puede convertirse en una evaluación sin influencia, debido a la falta de uso, mientras que la gestión del conocimiento sin evaluación podría orientar erróneamente la práctica del desarrollo al compartir conocimiento no validado (incluido el conocimiento que ha sido validado en un contexto específico pero que luego es compartido sin ninguna adaptación para su uso en un contexto diferente).

Las aplicaciones de gestión del conocimiento están surgiendo y es muy probable que su disponibilidad aumente exponencialmente. Esto está creando oportunidades para captar y compartir conocimientos a un ritmo cada vez mayor, con el consiguiente riesgo de utilización de conocimientos no validados para el contexto de la aplicación.

Para aclarar la cuestión de aplicar en un contexto específico el conocimiento generado por evaluaciones provenientes de otro contexto, una tabla booleana es una herramienta útil. Aunque el contexto tiene varias dimensiones, entre ellas el tiempo, el lugar, la escala, la cultura, las instituciones, la ecología, etc. (de hecho, las dimensiones pertinentes son específicas del contexto), a los efectos de la argumentación y la claridad de la presentación, vale la pena centrarse en tres dimensiones generales que muy probablemente serán relevantes para diferentes tipos de intervenciones de desarrollo: tiempo (¿cuándo se implementó la intervención de desarrollo?), lugar (¿dónde tuvo lugar la ejecución?) y escala (¿a qué escala se llevó a cabo la intervención?). Combinando las tres dimensiones habrá ocho situaciones, que se describen en la tabla $1 .^{7}$

La tabla 1 representa contextos iguales o diferentes con números 0 o 1 (una tabla binaria, que podría ampliarse para considerar los casos difusos con números entre 0 y 1). Para cada situación descrita en términos de tiempo, lugar y escala, la última columna indica los resultados asociados a la situación. Tomemos el siguiente ejemplo: en las zonas tribales del estado indio de Andhra Pradesh se ejecutó un proyecto de desarrollo rural entre los años 2002 y 2006, con una población objetivo de 3000 personas de las zonas tribales, y supuestamente una evaluación mostró que 2700 de esas personas pudieron salir de la pobreza gracias a las actividades ejecutadas por el proyecto.

7 Obsérvese que el número de situaciones es igual a $2^{a}$, donde $a$ es el número de dimensiones. Así, con cuatro dimensiones sería 16, y con cinco sería 32. Para captar la esencia del argumento bastan tres dimensiones, y el argumento sería más fuerte cuanto mayor fuera el número de dimensiones que se consideraran. 
Tabla 1. Tabla booleana que muestra diferentes situaciones en términos de tiempo, lugar y escala

\begin{tabular}{|c|c|c|c|c|}
\hline Situación & Tiempo & Lugar & Escala & Resultados \\
\hline $\mathrm{a}$ & 0 & 0 & 0 & R0 \\
\hline $\mathrm{b}$ & 0 & 0 & 1 & $?$ \\
\hline $\mathrm{c}$ & 0 & 1 & 0 & $?$ \\
\hline $\mathrm{d}$ & 0 & 1 & 1 & $?$ \\
\hline $\mathrm{e}$ & 1 & 0 & 0 & $?$ \\
\hline $\mathrm{f}$ & 1 & 0 & 1 & $?$ \\
\hline $\mathrm{g}$ & 1 & 1 & 0 & $?$ \\
\hline
\end{tabular}

¿Tiene sentido generalizar el conocimiento producido por esa evaluación para la misma población de Andhra Pradesh diez años después de la ejecución de ese proyecto? ¿O para las zonas tribales de algún otro estado de la India, o de otros países? ¿O para una población de treinta mil personas en el estado de Andhra Pradesh?

Para enmarcar la cuestión en términos más abstractos, utilizando la tabla 1, ¿tiene sentido extrapolar los resultados verificados en una situación (a), para un tiempo, lugar y escala específicos $\{0,0,0\}$, a otras situaciones en las que el tiempo, el lugar o la escala difieren (que se muestran en la tabla con 1, en lugar de 0)? ¿En qué condiciones o supuestos se justifica tal extrapolación?

En aras de la simplificación, el contexto en esta presentación se ha definido en términos de tiempo, lugar y escala y, como se indicó, el argumento sería más fuerte si se consideraran dimensiones adicionales de contexto. ¿Hay resultados sin contexto? ¿Mejores prácticas universales? ¿O los resultados dependen del contexto? La experiencia muestra que esto último es así y que cuanto más heterogéneos son los contextos, menos generalizables son los resultados (menor es su validez externa). En efecto, existe el riesgo de generalizaciones injustificadas.

En principio, cabe señalar que cuando se replican intervenciones en distintos lugares, y se obtienen resultados similares en contextos diferentes, se contribuye a aumentar la generalización de los resultados (su validez externa, VE). Pero no hay garantía de que en un nuevo contexto se mantengan los mismos resultados que en los contextos en los que se han realizado los ensayos (esto se indica en la tabla 1 con signos de interrogación). Sin embargo, la experiencia en un contexto específico puede proporcionar cierta orientación, o al menos un punto de partida para el diseño de intervenciones en un contexto diferente. No obstante, contrariamente a una opinión muy extendida, 
implícita en la frecuente referencia a lo que funciona, siempre es importante tener en cuenta el contexto. En realidad, el conocimiento generado por las evaluaciones no trata de lo que funciona sino de lo que funcionó en un contexto específico (o conjunto de contextos), y no debe darse por sentado que este conocimiento sobre el pasado, y en circunstancias específicas, se aplicará en el presente, especialmente si las circunstancias han cambiado. Estas cuestiones, y sus exploraciones filosóficas y metodológicas, están bien discutidas en Cartwright y Hardie (2012) y Pawson (2006).

Debe tenerse en cuenta que ni el éxito ni el fracaso en un contexto específico son evidencia suficiente de éxito o fracaso en un contexto diferente. Por lo tanto,

I. Si una intervención funcionó bien o tuvo éxito en un contexto específico, eso no significa que también funcionará bien en un contexto diferente (ya sea a una escala diferente o en un lugar diferente o en otro período). Por ejemplo, una intervención de microfinanzas puede tener éxito en una zona en la que hay un alto nivel de capital social, pero fracasar en un contexto en el que el capital social sea bajo.

II. Si una intervención fracasó en un contexto específico no significa que vaya a fracasar en otro contexto. Por ejemplo, si hay economías de escala, un piloto que fracasó en una escala baja puede tener éxito en una escala más alta (en la que, por ejemplo, puede justificarse la construcción de caminos rurales para facilitar el acceso a zonas remotas).

La gestión del conocimiento puede y debe desempeñar un papel fundamental en la codificación de los resultados, vinculándolos con las características del contexto (tiempo, lugar, escala) ${ }^{8}$ y haciendo que los resultados sean accesibles a los responsables políticos, los investigadores y la sociedad civil. En Dalkir (2011), Patton (2008) y Feinstein (2002) se muestran las formas en que esto puede hacerse. Las nuevas tecnologías, cada vez más disponibles, así como las nuevas aplicaciones, deberían facilitar este proceso para que la evaluación del desarrollo contribuya a mejorar la efectividad de las intervenciones.

Un reto importante para la gestión del conocimiento es identificar las características de las tres dimensiones que pueden aumentar el valor de desarrollo del conocimiento compartido. Por ejemplo, un aspecto importante es el área agroecológica de implementación de las intervenciones, como las zonas de alta montaña. Y, como se indica en este artículo, tales características son específicas del contexto. 


\section{Referencias}

Bamberger, M., Vaessen, J., y Raimondo, E. (2015). Dealing With Complexity in Development Evaluation: A Practical Approach. Nueva York: Sage.

Befani, B., Ramalingam, B., y Stern, E. (eds.). (2015) Towards Systemic Approaches to Evaluation and Impact, IDS Bulletin, 46(1).

Cartwright, N., y Hardie, J., (2012). Evidence-Based Policy. Oxford: OUP.

Dalkir, K. (2011). Knowledge Management in Theory and Practice. MIT Press.

Deaton, A. (2010). Instruments, randomization, and learning about development. Journal of Economic Perspectives, 48, 424-455.

Dervis, K. (2015). Disrupting development with digital technologies. Brookings Bloom Roundtable.

Feinstein, 0. (2015a). Getting to scale: how to bring development solutions to millions of poor people [reseña]. Development in Practice, 25, 289-290.

Feinstein, 0. (2015). Sobre el desarrollo de los sistemas de evaluación en América Latina y el Caribe. Revista del CLAD Reforma y Democracia, 62, 195-210.

Feinstein, 0. (2012). Evaluation as a Learning Tool. New Directions for Evaluation, 134, 103-112.

Feinstein, 0. (2009). National Evaluation Capacities: Lessons Learned and a Conceptual Scheme. National Evaluation Capacities. Nueva York: UNDP Independent Evaluation Office.

Feinstein, 0., y Beck, T. (2006). Evaluation of Development Interventions and Humanitarian Action. En F. Shaw, J. C. Greene, M. M. Mark (eds.), Handbook of Evaluation: Policies, Programs and Practices. Londres: Sage.

Feinstein, 0. (2002). Use of Evaluations and Evaluation of their Use. Evaluation, 8(4), 433-439.

Lemieux, V. (2015). We feel. En Big Data Observations of Citizen Sentiment about State Institutions and Social Inclusion Technical Knowledge Note. Washington DC: The World Bank Group.

Patton, M. Q. (2008). Utilization Focused Evaluation. Thousand Oakes: Sage.

Pawson, R. (2006). Evidence-based Policy. Londres: Sage.

Picciotto, R. (2014). The future of development evaluation. Revista de Evaluación de Programas y Políticas Públicas, 3, 259-287.

Picciotto, R. (2012). Experimentalism and development evaluation: will the bubble burst? Evaluation, 18(2), 213-229.

Ramalingam, B. (2013). Aid on the Edge of Chaos. Oxford: Oxford University Press.

Stern, E., Stame, N., Mayne, J., Forss, K., Davies, R., y Befani, B. (2012). Broadening the range of designs and methods for impact evaluation. DFID Working Paper. 